\title{
Registro de imágenes de rango basado en similaridad entre regiones
}

\author{
Range image registration by means similarity between regions \\ Manuel A. Maldonado ${ }^{1} \quad$ John W. Branch ${ }^{1} \quad$ German Sánchez T. ${ }^{2}$ \\ Recibido 21 de agosto de 2012, aceptado 28 de agosto de 2014 \\ Received: August 21, $2012 \quad$ Accepted: August 28, 2014
}

\begin{abstract}
RESUMEN
El registro de imágenes de rango es un paso intermedio en la construcción de modelos tridimensionales obtenidos mediante sensores de rango, debido principalmente a la naturaleza parcial del proceso de adquisición de los datos. Se propone un método de registro grueso de mallas triangulares para la reconstrucción de objetos tridimensionales, basado en la correspondencia de regiones de superficies empleando el Índice de Forma como descriptor principal de información topológica, y la métrica Earth Mover's Distance (EMD) como medida de similaridad entre regiones. Las principales ventajas de este método es que permite independencia de la prealineación inicial y transformación espacial, permitiendo obtener un registro adecuado sin importar la posición inicial de cada vista.
\end{abstract}

Palabras clave: Registro de imágenes, imágenes de rango, reconstrucción 3-D, índice de forma, Earth Mover's Distance.

\begin{abstract}
Range image registration is an intermediate step in the reconstruction of three-dimensional models process. Range sensors obtain a set of range images, and need to be registered mainly due to the partial nature of the acquisition process. We propose a rough registration method for triangular meshes based on matching regions using the Shape-Index like a topological descriptor, and the use of Earth Mover's Distance (EMD) metrics as a measure of similarity between regions. The main advantage of this method since it is independent of the initial pre-alignment and spatial transformation, it allows obtaining an adequate registration regardless of the original position of each view.
\end{abstract}

Keywords: Image registration, range images, 3-D reconstruction, shape index, Earth Mover's Distance.

\section{INTRODUCCIÓN}

El registro de imágenes de rango constituye una etapa inicial dentro de un proceso general denominado reconstrucción de formas tridimensionales (ver Figura 1). La obtención de un modelo computacional digital de una forma real inicia con la adquisición de los datos mediante dispositivos especializados denominados Escáner de Rango. Estos dispositivos generan imágenes construidas mediante las mediciones de profundidad desde el sensor hasta el objeto y son denominadas imágenes de rango.

Las imágenes de rango, como fuente de información para reconstruir superficies de objetos reales y cuya popularidad inició hace algo más de dos décadas con el trabajo de Hoppe [1], han seguido creciendo, principalmente debido al alto desarrollo de las tecnologías láser que brindan significativas mejoras en los niveles de precisión y flexibilidad

\footnotetext{
1 Departamento de Ciencias de la Computación y la Decisión. Universidad Nacional de Colombia. Medellín, Colombia. E-mail: mamaldon@unal.edu.co; jwbranch@unal.edu.co

2 Facultad de Ingenierías. Universidad del Magdalena. Cra 32 № 22-08. Edf. Docente, Cub. 3D401. Tel: (57) 54217940. Ext 1138. Santa Marta, Colombia. E-mail: gsanchez@unimagdalena.edu.co
} 
para la adquisición de datos de rango [2], lo que sugiere el uso en un amplio espectro de áreas en las que se requiera la representación digital de formas. De igual forma, otro aspecto que ha permitido la generalización del uso de este tipo de imágenes, lo constituye el hecho de que representan explícitamente la geometría de la superficie de la escena [3], por lo tanto, no poseen los problemas implícitos que se presentan en otros tipos de imágenes.

Debido a que el proceso de adquisición posee una naturaleza parcial, es necesario unificar el sistema coordenado de cada una de las imágenes adquiridas. Este proceso es realizado en una etapa denominada Registro, que consiste en llevar todas las imágenes adquiridas a un sistema de coordenadas común.

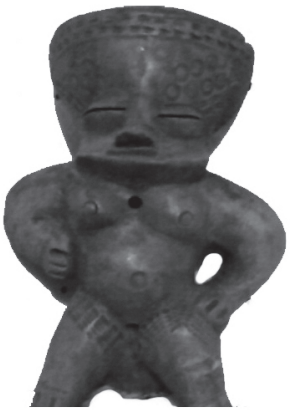

a)

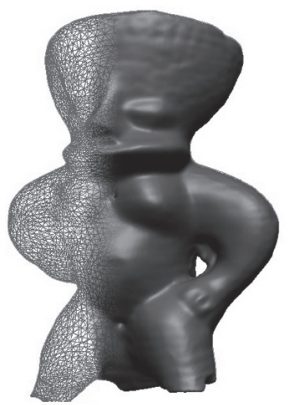

b)
Figura 1. Proceso de reconstrucción a) modelo original cerámico, b) modelo 3-D.

La clasificación de los métodos de registro es amplia y puede realizarse desde diferentes aspectos. Una clasificación común se realiza de acuerdo con el nivel de precisión en el que cada método une el par de imágenes. Así, puede existir registro grueso y registro fino. Es decir, si el objetivo es dar una alineación inicial o si el objetivo es unir con precisión las imágenes.

En este trabajo se propone un método de registro grueso, que utiliza el concepto de similaridad entre regiones para calcular las transformaciones geométricas necesarias para alinear correctamente las vistas parciales. A diferencia de los métodos clásicos que están basados en correspondencia de puntos, este método estima medidas de similaridad entre regiones.

\section{REVISIÓN DE LA LITERATURA}

El proceso de correspondencia de vistas parciales o imágenes generalmente es realizado mediante la búsqueda de correspondencias entre puntos asumiendo que existen áreas de solapamiento que permitan garantizar una solución. Bajo este supuesto se han propuesto diferentes variantes de este enfoque durante por lo menos las últimas dos décadas.

El método de registro más utilizado para alinear pares de imágenes es denominado ICP (Iterative Closest Point) [4]. Sin embargo, un número significativo de trabajos han seguido esta dirección intentando mejorar diferentes aspectos relacionados con el método; como su eficiencia, nivel de error, esquema de correspondencia, entre otros aspectos. Otros trabajos proponen variantes de alguna de las etapas del método clásico ICP, sobre la posición del sensor, identificación e indexado de características de la superficie y el uso de Least Median of Squares (LmedS), la cual busca minimizar la media error de la correspondencia [5-9].

En [10] se introduce el método Point-Signature para representar la estructura de un vecindario determinado. La correspondencia entre puntos se establece entre dos puntos con point signatures similares. El proceso de emparejamiento es rápido, pero requiere interpolar en algunas situaciones, lo que afecta la calidad. Es invariante a la rotación y traslación pero es sensible al ruido. En [11] se introduce el método de registro por PCA (Principal Component Analysis). Su objetivo es resumir un grupo amplio de variables en un nuevo conjunto más pequeño, sin perder una parte significativa de la información original. Se usa la dirección del eje principal de la nube de puntos para alinear una secuencia de imágenes de rango. Si la región de solapamiento es suficientemente grande, los ejes de las imágenes deben coincidir, de modo que, aplicando una sola transformación, se alineen los ejes, y por ende, las imágenes. Este método implica el cálculo de la matriz de covarianzas de cada imagen, lo que implica una alta velocidad y precisión con suficientes puntos. En [12] se propone un método de cálculo de la correspondencia entre imágenes a partir de líneas rectas. El algoritmo fue aplicado al registro de estructuras con regiones planas como edificios, y obtiene buenos resultados, aunque la dificultad reside en que el método no es automático, además está limitado por la geometría de las vistas.

El Registro por Algoritmos Genéticos (AG) [13] consiste en la búsqueda de un individuo cuyo 
cromosoma está compuesto por 6 parámetros que determinan la transformación rígida que alinea un par de imágenes: 3 elementos del vector de traslación y los 3 ángulos de la rotación. Las variaciones de este método se presentan alrededor de la función de aptitud del AG. El método es robusto frente al ruido, mínimo locales y datos atípicos, aunque, como consecuencia de las técnicas evolutivas, requiere un tiempo de cómputo muy alto, y dependiendo de la función de aptitud, el costo del cálculo de correspondencias puede llegar a ser muy alto. En [14] proponen un método que se basa en la diferencia de puntos caracterizados por sus curvaturas principales como característica geométrica para el proceso de emparejamiento. Este método solo requiere una correspondencia para el cálculo de la transformada. El método calcula las curvaturas $\left(\mathrm{k}_{1}, \mathrm{k}_{2}\right)$ en un punto, donde $\mathrm{k}_{1}$ y $\mathrm{k}_{2}$ corresponden a la curvatura máxima y mínima de la superficie en el punto dado. Adicionalmente, se considera el vector normal y la dirección de la curvatura principal. Aunque no siempre es posible calcular las curvaturas y direcciones en un punto, se propone una superficie parametrizada. El mayor problema del método es que no es robusto, el algoritmo se detiene cuando encuentra una correspondencia buena, presentando posibles falsos positivos.

Las principales debilidades de los métodos de registro se centran en el aspecto realizado a la correspondencia, es decir, la métrica estipulada para determinar si dos puntos de diferentes imágenes constituyen el mismo punto, o el más cercano, en el objeto tratado. Por tal razón el enfoque propuesto consiste en determinar la correspondencia entre puntos, considerando la geometría de la región que los rodea.

\section{MÉTODO DE CORRESPONDENCIA}

El método propuesto consiste en alinear vistas parciales a partir de las características geométricas descritas por el índice de forma calculado en cada vértice utilizando las estimaciones de curvatura. Un grupo de 3 vértices son seleccionados y en ellos se estima un histograma de las medidas de los índices de forma del vecindario que rodea cada vértice. Estos son emparejados con un grupo del mismo tamaño en la vista a registrar de acuerdo con su similaridad utilizando EMD como métrica. Una vez seleccionadas las parejas de vértices se procede a estimar la transformación geométrica que acerca dichos conjuntos de puntos y se cuantifica el resultado de acuerdo con la estimación de la métrica de interpenetración. A continuación se describe cada paso.

\section{Cálculo del índice de forma}

Como se mencionó, para el cálculo del índice de forma se requiere una estimación de las curvaturas principales por cada vértice. La curvatura es una medida invariante, ya que permanecen constantes ante traslaciones y rotaciones, a excepción de cambios de escala.

La curvatura mide el cambio de dirección del vector tangente a una curva, el cual corresponde al inverso del radio de la esfera tangente a la superficie. Se obtiene a partir de la Segunda Forma Fundamental, la cual representa la variación de la normal de punto a punto. Para una superficie continua se cumple:

$$
k_{n}=\left(\begin{array}{ll}
s & t
\end{array}\right)\left(\begin{array}{ll}
e & f \\
f & g
\end{array}\right)\left(\begin{array}{l}
s \\
t
\end{array}\right)=\left(\begin{array}{ll}
s & t
\end{array}\right) I I\left(\begin{array}{l}
s \\
t
\end{array}\right)
$$

para cualquier vector $(s, t)$ en el plano tangente (expresado en términos de un sistema de coordenadas ortonormal centrado en el punto). La matriz II es conocida como matriz Weingarten o Segunda Forma Fundamental, la cual puede ser diagonalizada para obtener:

$$
k_{n}=\left(\begin{array}{ll}
s^{\prime} & t^{\prime}
\end{array}\right)\left(\begin{array}{cc}
k_{2} & 0 \\
0 & k_{2}
\end{array}\right)\left(\begin{array}{l}
s^{\prime} \\
t^{\prime}
\end{array}\right)=k_{2} s^{\prime 2}+k_{2} t^{\prime \prime 2}
$$

El máximo y el mínimo valor de la curvatura normal en un punto $p$ de la superficie $S$ son llamadas curvaturas principales [15] de $S$ en $p$, denotadas por $k_{1}$ y $k_{2}$. Los vectores $\left(s^{\prime}, t^{\prime}\right)$ en los cuales se encuentran los valores extremos son llamadas direcciones principales.

La matriz II está definida en función de las derivadas direccionales sobre la superficie:

$$
I I=\left(\begin{array}{cc}
D_{u} n & D_{v} n
\end{array}\right)=\left(\begin{array}{ll}
\frac{\partial n}{\partial u} \cdot u & \frac{\partial n}{\partial v} \cdot u \\
\frac{\partial n}{\partial u} \cdot v & \frac{\partial n}{\partial v} \cdot v
\end{array}\right)
$$


donde $(u, v)$ son las direcciones del sistema de coordenadas ortonormal en el punto. Multiplicando II por un vector del plano tangente se obtiene la derivada de la normal en esa dirección:

$$
\text { IIs }=D_{s} n
$$

Aunque esta expresión está definida para superficies continuas, puede realizarse una aproximación para el caso discreto utilizando diferencias finitas: para un triángulo con la dirección de sus aristas definidas, tenemos las diferencias de normales en dichas direcciones (ver Figura 2).

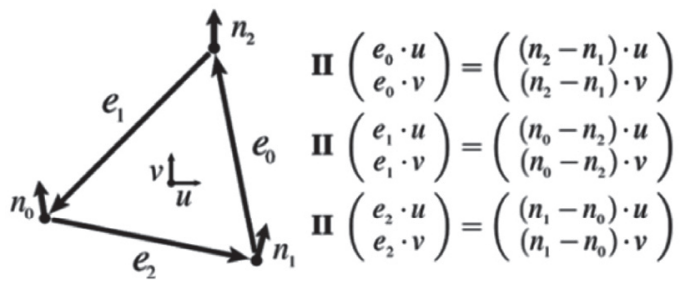

Figura 2. Diferencia de normales por cara.

Esto ofrece un conjunto de restricciones lineales para el cálculo de II utilizando mínimos cuadrados. Una vez resuelto el sistema, tenemos las curvaturas principales por vértice.

Una vez obtenidas las curvaturas, se puede obtener el índice de forma asociado. El índice de forma fue propuesto en [16], y es una medida física angular que captura la noción intuitiva de forma local en un punto de la superficie:

$$
s=\frac{2}{\pi} \arctan \frac{k_{2}+k_{1}}{k_{2}-k_{1}}
$$

El rango del índice varía entre -1 y 1 . Un punto de una superficie convexa con igual valor de curvaturas principales tiene un índice de la forma de 1 . Un punto de una superficie cóncava con igual valor de curvaturas principales tiene un índice de la forma de -1 . Un punto silla con igual magnitud de curvaturas principales pero signos distintos tiene un índice de la forma de 0 .

Búsqueda de vértices geométricamente similares Una vez obtenidos los índices de forma por vértice de cada vista, i) se seleccionan manualmente al menos 3 vértices en la primera vista, ii) se genera un histograma con los índices de forma del vecindario alrededor de cada vértice, y iii) se realiza una búsqueda de los vértices de la segunda vista que tengan histogramas similares.

Un histograma representa la frecuencia de valores de una variable. La variable cuantificada es categórica, es decir, sus valores son categorías o etiquetas alfanuméricas, y la cantidad de posibles valores es finita. Cada valor representado en el histograma corresponde a una clase (bin). Por lo general, la cantidad de clases del histograma es igual a la cantidad de valores de la variable evaluada. Pero en este caso, el índice de forma es una variable continua, por lo que no es posible representarla directamente en un histograma, por lo tanto, agrupamos sus posibles valores en subintervalos. La cantidad de subintervalos utilizados es de 10 , es decir, se dividió el intervalo $[-1,1]$ en 10 subintervalos, cada uno asociado a una clase del histograma.

Dados dos histogramas, se requiere una medida cuantitativa de su diferencia que refleje la percepción de similitud lo mejor posible. En [17] se propone la métrica EMD (Earth Mover's Distance) para establecer la diferencia entre dos distribuciones, la cual consiste en calcular la mínima cantidad de redistribución necesaria para convertir una distribución en la otra. El EMD es calculado utilizando un algoritmo de optimización lineal basado en la solución al problema de transporte [18-19], el cual es un caso particular de problema de programación lineal en el cual se debe minimizar el coste del abastecimiento de una serie de puntos de demanda (destino), a partir de un grupo de puntos de oferta (origen).

\section{Cálculo de similitud entre histogramas}

Para el cálculo de la similitud entre histogramas, se utiliza la métrica EMD, la cual se define como el costo mínimo para transformar un histograma en otro, donde hay una distancia de suelo entre las características representadas en el histograma. Dados dos histogramas P, Q se define EMD como:

$$
\operatorname{EMD}(P, Q)=\frac{\left(\min _{f_{i j}} \sum_{i, j} f_{i j} d_{i j}\right)}{\sum_{i, j} f_{i j} d_{i j}}
$$


Sujeto a:

$$
\begin{aligned}
& f_{i j}>0 ; \quad \sum_{i, j} f_{i j} \leq p_{i} ; \quad \sum_{i, j} f_{i j} \leq Q_{j} \\
& \sum_{i, j} f_{i j} d_{i j}=\min \left(\sum_{i} P_{i}, \sum_{j} Q_{j}\right)
\end{aligned}
$$

Donde $f_{i j}$ denota los flujos de red asociados al problema del transporte, $f_{i j}$ representa la cantidad transportada desde la clase $i$ de $P$ a la clase $j$ de $Q$, y $d_{i j}$ la distancia de suelo entre la clase $i$ y la clase $j$. En este trabajo se utiliza la variante $\overline{E M D}$ propuesta en [20]:

$$
\overline{E M D}(P, Q)=\left(\min _{i, j} \sum_{i, j} f_{i j} d_{i j}\right)+\left|\sum_{i} P_{i}-\sum_{j} Q_{j}\right| \alpha \max d_{i j}
$$

$\overline{E M D}$ es una métrica definida para dos histogramas, sujeto a las restricciones de la ecuación (7).

\section{Cálculo de error e índice de interpenetración de superficies}

El índice de interpenetración de superficies (SIMSurface Interpenetration Measure) está basado en el efecto natural resultante del registro de superficies, debido a pequeñas distorsiones locales causadas por las limitaciones en la precisión del sensor, o por ruido. Cuantificando la interpenetración se puede establecer una evaluación más precisa, y es posible ofrecer un control más robusto del registro, aunque en este trabajo solo se utiliza como una medida para evaluar la calidad del registro. Un valor alto de SIM representa un buen registro, y de igual manera, malas alineaciones producen valores bajos.

Después de la alineación de dos vistas, $A$ y $B$, se realiza la identificación de los puntos de interpenetración en $A$ respecto de $B$, los cuales llamaremos $C_{A B}$. Para cada punto $\mathrm{p} \in \mathrm{A}$, se define un vecindario $\mathrm{N}_{\mathrm{p}}$ de radio $n$ centrado en $p$. Con $q$ definido como un punto en el vecindario $N_{p}, \mathrm{y} c$ como el punto correspondiente de $p$ en $B$, obtenido por medio de una búsqueda de puntos cercanos utilizando un $k d$-tree [22], y $n_{c}$ el vector normal en $c$. Por lo tanto, el conjunto de puntos de interpenetración en $A$ está dado por:

$$
C_{A, B}=\left\{p \in A /\left|\left(\overrightarrow{q_{i}-c} \cdot \overrightarrow{n_{c}}\right)\left(\overrightarrow{q_{j}-c} \cdot \overrightarrow{n_{c}}\right)\right|<0\right\}
$$

Donde $q_{i}, q_{j} \in N_{p} y i \neq j$. Los puntos $C_{A, B}$, corresponden a los puntos en $A$ cuyos vecindarios incluyen al menos un par de puntos separados por un plano tangente local, obtenido a partir de los puntos correspondientes en $B$, como se ve en la Figura 3 .

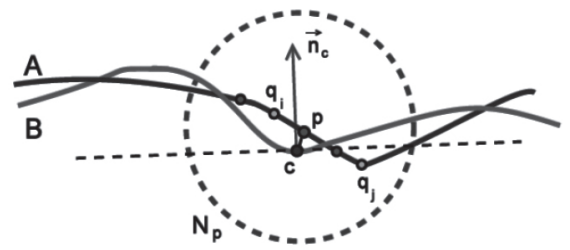

Figura 3. Punto de interpenetración p en A respecto de B [21].

\section{RESULTADOS}

En esta sección se presentan los resultados de algunos métodos de otros autores y el método propuesto en este trabajo. Para la comparación se realizó el registro de las vistas parciales de un par de imágenes: el conejo y el armadillo de Stanford [23]. Los resultados de los otros métodos fueron obtenidos utilizando el toolbox de registro publicado por Salvi [24], ejecutado en MATLAB R2010a sobre un procesador Intel Core 2 Duo de 3.00 GHz. La implementación del método propuesto fue realizada en .NET 2.0 C\\#/C++ y cuenta con un visor en OpenGL.

La Figura 4 muestra la selección de los vecindarios sobre $S_{1}$. La búsqueda de los vértices $\left\{\mathrm{v}_{2 \mathrm{i}}\right\}$ sobre $S_{2}$ se realiza calculando el valor de $\overline{E M D}$ entre cada alrededor de $\left\{v_{1 \mathrm{i}}\right\}$ y los vecindarios de radio $\mathrm{r}$ alrededor de cada vértice de $S_{2}$, obteniendo un listado de distancias por vecindario de $S_{1}$. De los listados obtenidos se escoge un vértice de cada

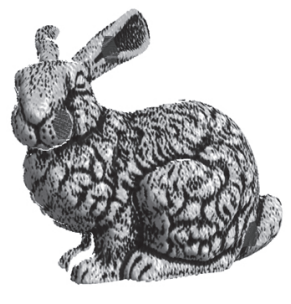

(a)

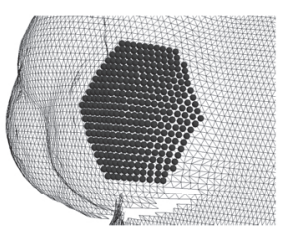

(b)
Figura 4. Vista parcial del conejo: (a) Mapa de color del índice de forma, (b) Vecindario de radio 10 alrededor del vértice seleccionado. 
lista, y se realiza una alineación contra los vértices seleccionados de $S_{1}$. De esta búsqueda, se escoge la combinación de vértices de $S_{2}$ con el menor error de alineación respecto de $S_{1}$. La Figura 5 y 6 muestran el resultado del proceso de alineación de dos vistas de diferentes objetos.

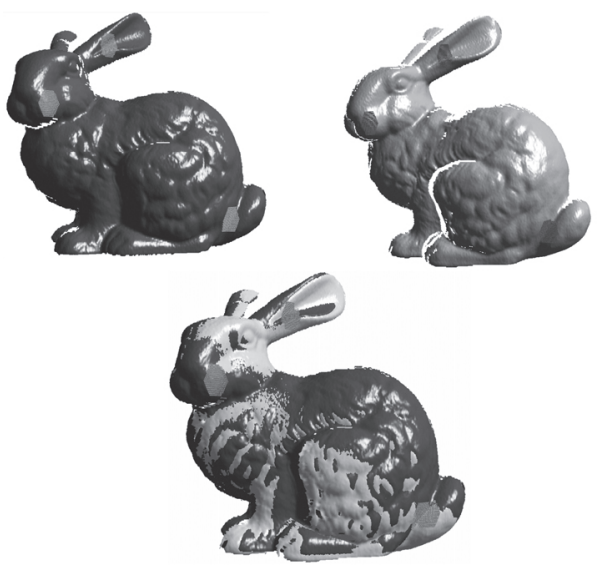

Figura 5. Vecindarios seleccionados: (a) Vecindarios seleccionados de S1, (b) Vecindarios similares de $\mathrm{S} 2$, y (c) el resultado de la alineación.

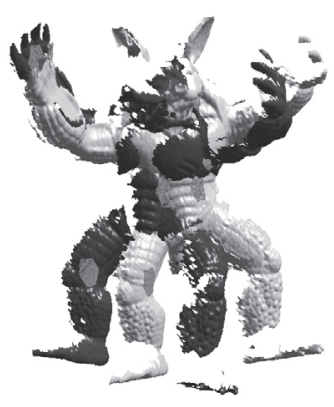

(a)

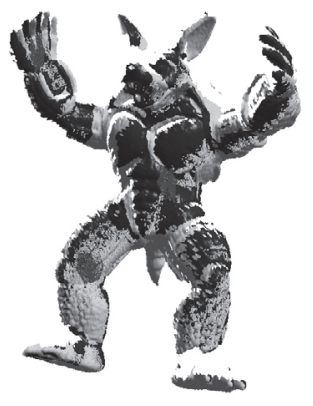

(b)
Figura 6. Vecindarios seleccionados: (a) Vecindarios seleccionados de $\mathrm{S} 1$, (b) el resultado de la alineación.

La Figura 7 muestra el comportamiento de la minimización del error en la búsqueda de los pares de vértices en los vecindarios. El error reportado es una lista ordenada del promedio de las distancias entre los pares de vértices más cercanos seleccionados con un kd-tree.

Una comparación del nivel de error, estimado como el promedio de las distancias de los vértices

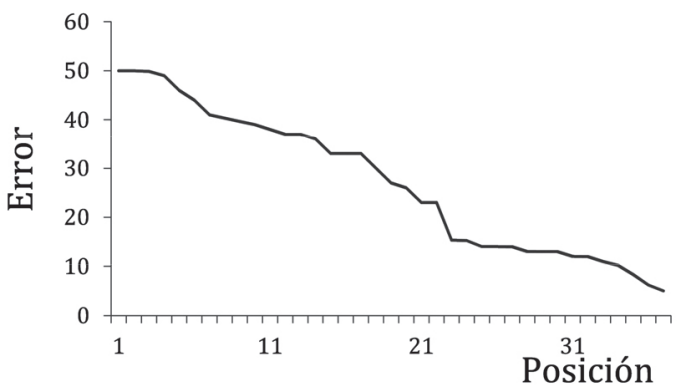

Figura 7. Comportamiento del error en el proceso de búsqueda de parejas de vértices.

del área de solapamiento con su más cercano, del método propuesto con otros métodos reportados en la literatura usando los objetos Bunny y Armadillo, se muestra en la Tabla 1.

Tabla 1. Comparación con diferentes métodos.

\begin{tabular}{|l|c|c|}
\hline \multicolumn{1}{|c|}{ Método } & $\begin{array}{c}\text { Bunny } \\
\text { (Error) }\end{array}$ & $\begin{array}{c}\text { Armadillo } \\
\text { (Error) }\end{array}$ \\
\hline PCA [11] & 47,2429 & 49,9713 \\
\hline Besl-McKay [4] & 2,7731 & 4,3491 \\
\hline $\begin{array}{l}\text { Chen-Mendioni } \\
\text { [25] }\end{array}$ & 2,8631 & 1,2852 \\
\hline Jost [26] & 7,9114 & 4,7693 \\
\hline Método propuesto & 1,5380 & 2,8610 \\
\hline
\end{tabular}

\section{CONCLUSIONES}

El registro o alineamiento de imágenes de rango basado en correspondencia de regiones mostró ser un enfoque que regenera registros iniciales con precisión. El alineamiento generado está guiado por dos métricas que permiten acercar las imágenes correctamente e integrarlas. La primera es la similitud entre regiones, acerca las vistas correspondientes y la segunda, el índice de interpenetración, permite que se integren.

Un requerimiento del método es la necesidad de la existencia de un prealineamiento. Este requerimiento no constituye una limitación debido a que los dispositivos de adquisición modernos generan mallas cercanas entre sí. En este sentido, el método es determinístico lo que sugiere un uso eficiente en el uso de recursos computacionales frente a las debilidades de los esquemas estocásticos, como los basados en algoritmos genéticos. El método permite alinear pares de imágenes con estados de rotación y translación arbitrarios. 
El problema de las múltiples resoluciones en la densidad de las mallas, no fue considerado, el método asume que la adquisición se realizó con las mismas configuraciones iniciales en el dispositivo de adquisición. Una directriz en este sentido puede ser adicionar una etapa de simplificación de puntos a las mallas iniciales para aproximar las densidades de estas previo al alineamiento. De igual forma, la variación en la escala no fue abordada en esta propuesta, en este sentido, como trabajo futuro se propone utilizar una medida de densidad para estimar el factor de escala que se aplique a las diferentes vistas.

\section{REFERENCIAS}

[1] H. Hoppe, T. DeRose, T. Duchamp, J. McDonald and W. Stuetzle. "Surface reconstruction from unorganized points". SIGGRAPH Comput. Graph. Vol. 26, Issue 2, pp. 71-78. July, 1992. ISSN: 0097-8930. DOI: $10.1145 / 142920.134011$

[2] F. Forster. "A high-resolution and high accuracy real-time $3 \mathrm{~d}$ sensor based on structured light". 3D Data Processing, Visualization, and Transmission. Third International Symposium. Chapel Hill, USA. June 14-16, 2006.

[3] J.W. Branch, F. Prieto and P. Boulanger. "Automatic extraction of a quadrilateral network of NURBS patches from range data using evolutionary strategies". Lecture Notes in Computer Science. Vol. 4842, pp. 701-710. November, 2007. ISSN: 0302-9743. DOI: 10.1007/978-3-540-76856-2_69.

[4] P. Besl and N. McKay. "A Method for Registration of 3-D Shapes". IEEE Trans. Pattern Analysis and Machine Intelligence. Vol. 14, Issue 2, pp. 239-256. February, 1992. ISSN: 0162-8828. DOI: 10.1109/34.121791.

[5] Z. Zhang. "Iterative Point Matching for Registration of Free-form Curves and Surfaces”. Int. Jour. Computer Vision. Vol. 13, Issue 2, pp. 119-152. 1994. ISSN: 1573-1405. DOI: 10.1007/BF01427149.

[6] R. Bergevin, M. Soucy, H. Gagnon and D. Laurendeau. "Towards a General MultiView Registration Technique". IEEE Trans. Pattern Analysis and Machine Intelligence. Vol. 18, Issue 5, pp. 540-547. May, 1996. ISSN: 0162-8828. DOI: 10.1109/34.494643.
[7] C. Dorai, J. Weng and A. Jain. "Optimal Registration of Multiple Range Views". Int. Conf. on Pattern Recognition. Washington, USA. October, 1994.

[8] O. Carmichael and M. Hebert. "Unconstrained Registration of Large 3D Point Sets for Complex Model Building”. IEEE Int. Conf. on Intelligent Robots and Systems. Victoria, Canada. October, 1998.

[9] O. Faugeras. "The Representation, Recognition and Locating of 3-D Objects". Int. J. Robotic Res. Vol. 5, Issue 3, pp. 27-52. July, 1986. ISSN: 0278-3649. DOI: 10.1177/027836498600500302.

[10] C.J.R. Chua. "Point signatures: a new representation for $3 \mathrm{~d}$ object recognition". Int. J. Comput. Vision. Vol. 25, Issue 1, pp. 63-85. October, 1997. ISSN: 0920-5691. DOI: 10.1023/A:1007981719186.

[11] D. Chung and Y. Lee. "Registration of multiplerange views using the reverse-calibration technique". Pattern Recogn. Vol. 31, Issue 4, pp. 457-464. June, 1998. ISSN: 0031-3203. DOI: 10.1016/S0031-3203(97)00063-0.

[12] I. Stamos and M. Leordeanu. "Automated feature-based range registration of urban scenes of large scale". IEEE Computer Society Conference on Computer Vision and Pattern Recognition. Vol. 2, pp. 555-561. June 18-20, 2003. ISSN: 1063-6919. DOI: 10.1109/CVPR.2003.1211516.

[13] K. Brunnstrom and A. Stoddart. "Genetic algorithms for free-form surface matching". In International Conference of Pattern Recognition. Vienna, Austria. August 25-29, 1996.

[14] J. Feldmar and N. Ayache. "Rigid and affine registration of smooth surfaces using differential properties". Lecture Notes in Computer Science. Vol. 801, pp. 396-406. July 31, 1994. ISSN: 0302-9743. DOI: 10.1007/BFb0028371.

[15] S. Rusinkiewicz. "Estimating curvatures and their derivatives on triangle meshes". In Symposiumon 3D Data Processing, Visualization, and Transmission. Thessaloniki, Greece. September 6-9, 2004.

[16] J.J. Koenderink and A.J. van Doorn. "Surface shape and curvature scales". Image Vision Comput. Vol. 10, Issue 8, pp. 557-565. Butterworth, USA. 
October, 1992. ISSN: 0262-8856. DOI: 10.1016/0262-8856(92)90076-F.

[17] Y. Rubner, C. Tomasi and L.J. Guibas. "A metric for distributions with applications to image databases". In Proceedings of the Sixth International Conference on Computer Vision. Washington, USA. June 4-7, 1998. ISBN: 81-7319-221-9. DOI: 10.1109/ ICCV.1998.710701.

[18] S.T. Rachev. "The monge-Kantorovich Mass transference problem and its stochastic applications". Theory of Probability and its Applications. Vol. 29, Issue 4, pp. 647-676. 1985. ISSN: 1095-7219. DOI: 10.1137/1129093.

[19] F.L. Hitchcock. "The distribution of a product from several sources to numerous localities". Journal of Math. Phys. Vol. 20, pp. 224-230. 1941.

[20] O. Pele and M. Werman. "A linear time histogram metric for improved sift matching". European Conference on Computer Vision, ECCV. Marseille, France. 2008.

[21] L. Silva, O. Bellon and K. Boyer. "Precision range image registration using a robust surface interpenetration measure and enhanced genetical gorithms". Journal IEEE
Transactions on Pattern Analysis and Machine Intelligence. Vol. 27, Issue 5, pp. 762-776. May, 2005.

[22] J.L. Bentley. "Multidimensional binary search trees used for associative searching". Commun ACM. Vol. 18, Issue 9, pp. 509517. September, 1975. ISSN: 0001-0782. DOI: $10.1145 / 361002.361007$.

[23] G. Turk. "The Stanford bunny". January, 2000. Date of visit: May 14, 2012. URL: https://graphics.stanford.edu/data/3Dscanrep/

[24] J. Salvi, C. Matabosch, D. Fofi and J. Forest. "A review of recent range image registration methods with accuracy evaluation". Image and Vision Computing. Vol. 25, Issue 5, pp. 578-596. May, 2007. ISSN: 0262-8856. DOI: 10.1016/j.imavis.2006.05.012.

[25] Y. Chen and G. Medioni. "Object modeling by registration of multiple range images". IEEE International Conference on Robotics and Automation. Sacramento, Canada. April 9-11, 1991.

[26] T. Jost and H. Hugli. "A multi-resolution scheme ICP algorithm for fast shape registration". Padova, Italy. June 19-21, 2002. 